\title{
Prediction of bleeding etiology: The clinician is vindicated!
}

\author{
Stephen E Congly MD FRCPC, Alexander I Aspinall MD PhD FRCPC
}

G astrointestinal (GI) bleeding is associated with significant morbidity and mortality. Variceal bleeds are significantly more lifethreatening than nonvariceal bleeds, with mortality rates of $18 \%$ in variceal bleeds (1) versus $5 \%$ in nonvariceal bleeds (2). Determining the acuity of the bleed is important to ensure that therapeutic upper endoscopy is performed within a window of time that balances mortality risk against optimal use of a limited resource. Several prediction scores have been proposed to risk stratify patients with upper GI bleeds, including the Rockford and Blatchford scales $(3,4)$. These scores are, however, limited because they do not discriminate between GI bleeds of variceal and nonvariceal origin. This is a crucial distinction because management is different.

In the current issue of the Canadian Journal of Gastroenterology, Alharbi et al (5) (pages 187-192) investigate preprocedure characteristics of patients ultimately diagnosed with variceal and nonvariceal upper GI bleeds. The patients were enrolled in the REgistry of patients undergoing endoscopic and/or Acid Suppression therapy and Outcomes analysis for upper gastrointestinal bleediNg (REASON) trial, with an aim to identify patient characteristics that distinguish variceal bleeds from nonvariceal bleeds. This multicentre, Canadian study conducted over an 18 -month period found $11 \%$ of upper GI hemorrhages to be secondary to varices, which is similar to the rate found in the United Kingdom (6). Thirty-day mortality rates were significant for nonvariceal bleeds $(9.4 \%)$ and $14.4 \%$ for variceal bleeds. After multivariate analysis, statistically significant risk factors associated with a variceal bleed included a history of liver disease, the presence of any stigmata of liver disease, alcohol abuse, hematemesis and hematochezia. This study also provides a unique snapshot of the management of GI bleeds in a very large geographical area. While outcome comparisons among centres were not reported, as a whole, mortality figures were similar to those reported in other western societies. Somewhat surprisingly, in $42 \%$ of cases, a rectal examination appeared not to be performed. In 19\% of cases, a fecal occult blood test from a stool sample was performed - a test that has no utility in the context of an upper GI bleed (however, these fecal occult blood tests may have been performed by nongastroenterologists).

Given that definitive management of variceal and nonvariceal upper GI bleeds is different, as are the transfusion goals and adjunct therapy (albumin and antibiotics in variceal bleeding), better methodology to discriminate variceal from nonvariceal bleeding has the potential to improve patient outcomes through earlier recognition. Previous work has investigated predictive factors of who will experience poor outcomes with a GI bleed (ie, the Rockford and Blatchford scales). These scales have been validated in real-world populations of patients presenting with both variceal and nonvariceal bleeding $(3,4)$.

An extension of this concept is to predict which patients are likely to experience variceal bleeds before a potentially life-threatening bleeding episode. This includes measuring liver stiffness through transient elastography (7) and biochemical parameters such as a platelet to spleen ratio $(8,9)$. Unfortunately, a diagnosis of cirrhosis is often made at the time of a variceal bleed, limiting the utility of these techniques, and emphasizing a need for a high index of suspicion for the presence of high-risk varices in outpatient populations. It would be of clinical relevance to incorporate the significant findings of the study by Alharbi et al in an outpatient model of at-risk patients who have yet to experience a variceal bleed.

Two single-centre trials from Thailand have also studied how to differentiate nonvariceal bleeds from variceal GI bleeds. Pongprasobchai et al (10) found an 18\% incidence of variceal bleeding in their study, which is higher than the rate found by the REASON investigators. Factors supporting a variceal source include a history of cirrhosis, signs of chronic liver disease, hematemesis and blood from a nasogastric aspirate. Further support was found in the recent study by Rattanasupar (11), which confirmed the predictive value of signs and symptoms of chronic liver disease, as well as a history of cirrhosis. Interestingly, the type of emesis did not appear to be predictive; rather, a low hematocrit of $<30 \%$ was found to have a significant association with varices. In combination, this study found that all three tests had a specificity of $98.2 \%$, but a poor sensitivity of $59.5 \%$. The best test characteristics were found with clinical signs of chronic liver disease. It is difficult to extrapolate these findings to the Canadian population given the different demographics of the Thai population and a likely higher proportion of patients with cirrhosis due to viral causes (hepatitis B in particular).

The study by Alharbi et al provides us with the largest series investigating a mixed population of patients presenting with upper GI hemorrhage. It confirms some preprocedure patient characteristics that can predict a greater likelihood of GI bleeding due to varices. While consistent, these characteristics are not terribly surprising; however, an important take home message is to have a high index of suspicion for a variceal source of bleeding in patients with a known history of cirrhosis or with clinical signs of chronic liver disease.

Overall, the study by Alharbi et al vindicates bedside examination, confirming that clinical clues, obtained through history and physical examination, can accurately predict which patients are likely to experience upper GI bleeding due to varices. These factors in the clinical assessment should be used in addition to using risk prediction scores such as the Rockford or Blatchford scale. Further validation of predictive factors in a prospective trial, as well as in other countries, might help in the development of a clinical predictive scoring system to be used in combination with history and physical examination.

DISLCOSURE: The authors have no financial disclosures or conflicts of interest to declare.

\section{REFERENCES}

1. Chalasani N, Kahi C, Francois F, et al. Improved patient survival after acute variceal bleeding: A multicenter, cohort study. Am J Gastroenterol 2003;98:653-9.

2. Barkun A, Sabbah S, Enns R, et al. The Canadian Registry on Nonvariceal Upper Gastrointestinal Bleeding and Endoscopy (RUGBE): Endoscopic hemostasis and proton pump inhibition are associated with improved outcomes in a real-life setting. Am J Gastroenterol 2004;99:1238-46. 
3. Stanley AJ, Ashley D, Dalton HR, et al. Outpatient management of patients with low-risk upper-gastrointestinal haemorrhage: Multicentre validation and prospective evaluation. Lancet 2009;373:42-7.

4. Lahiff C, Shields W, Cretu I, et al. Upper gastrointestinal bleeding: Predictors of risk in a mixed patient group including variceal and nonvariceal haemorrhage. Eur J Gastroenterol Hepatol 2012;24:149-54.

5. Alharbi A, Almadi M, Barkun A, Martel M; the REASON investigators. Predictors of a variceal source among patients presenting with upper gastrointestinal bleeding.

Can J Gastroenterol 2012;26:187-92.

6. Hearnshaw SA, Logan RF, Lowe D, Travis SP, Murphy MF, Palmer KR. Acute upper gastrointestinal bleeding in the UK: Patient characteristics, diagnoses and outcomes in the 2007 UK audit. Gut 2011;60:1327-35.
7. Sporea I, Ratiu I, Sirli R, Popescu A, Bota S. Value of transient elastography for the prediction of variceal bleeding. World J Gastroenterol 2011;17:2206-10.

8. Sarangapani A, Shanmugam C, Kalyanasundaram M, Rangachari B, Thangavelu P, Subbarayan JK. Noninvasive prediction of large esophageal varices in chronic liver disease patients. Saudi J Gastroenterol 2010;16:38-42

9. Mattos AZ, Mattos AA, Vianna FF, Musskopf MI, Pereira-Lima JC, Maciel AC. Platelet count/spleen diameter ratio: Analysis of its capacity as a predictor of the existence of esophageal varices. Arq Gastroenterol 2010;47:275-8.

10. Pongprasobchai S, Nimitvilai S, Chasawat J, Manatsathit S. Upper gastrointestinal bleeding etiology score for predicting variceal and non-variceal bleeding. World J Gastroenterol 2009;15:1099-104.

11. Rattanasupar A. Role of clinical parameters for predicting the cause of upper gastrointestinal bleeding. J Med Assoc Thai 2012;95:22-8. 


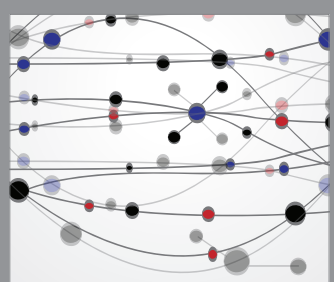

The Scientific World Journal
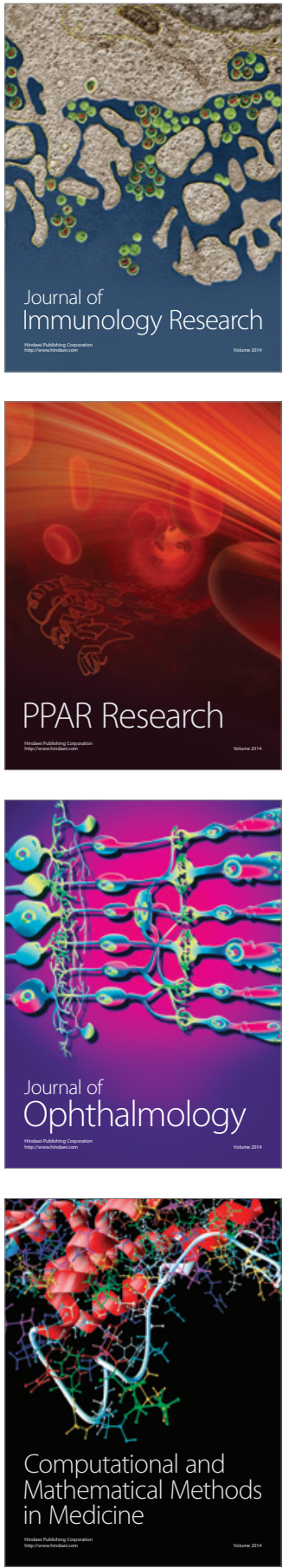

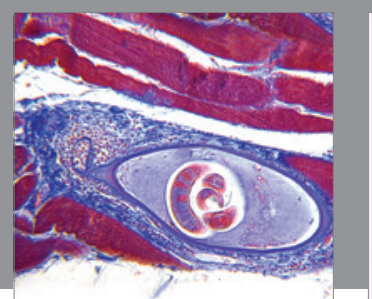

Gastroenterology Research and Practice

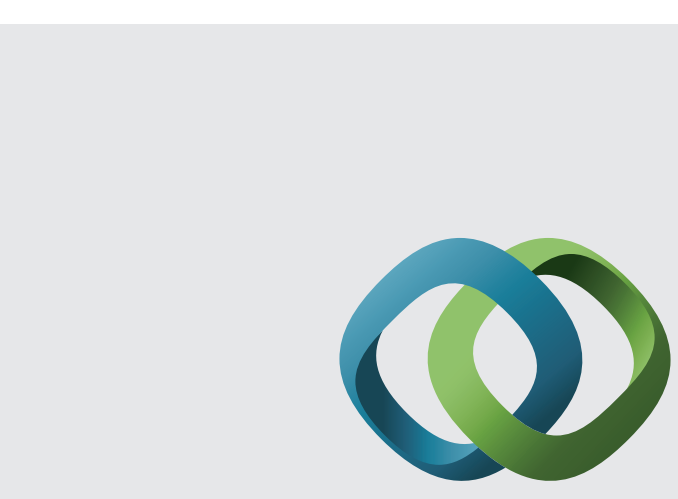

\section{Hindawi}

Submit your manuscripts at

http://www.hindawi.com
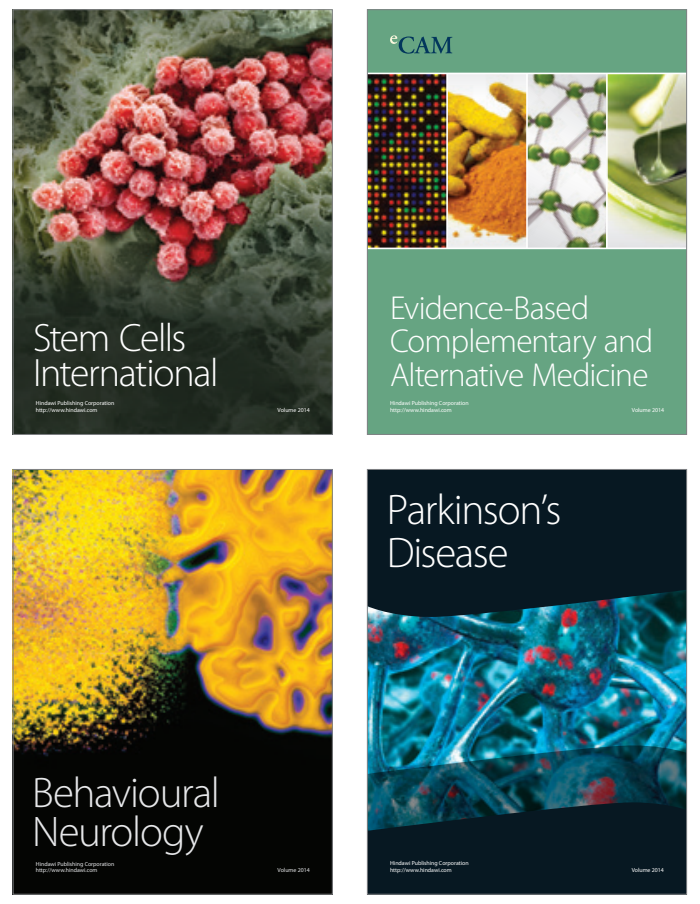
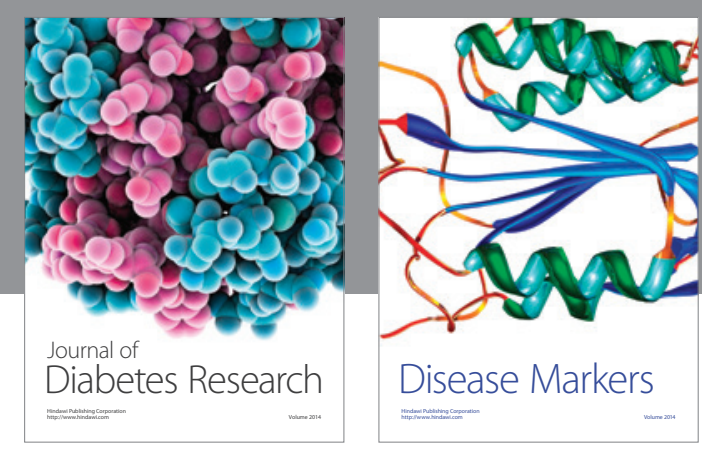

Disease Markers
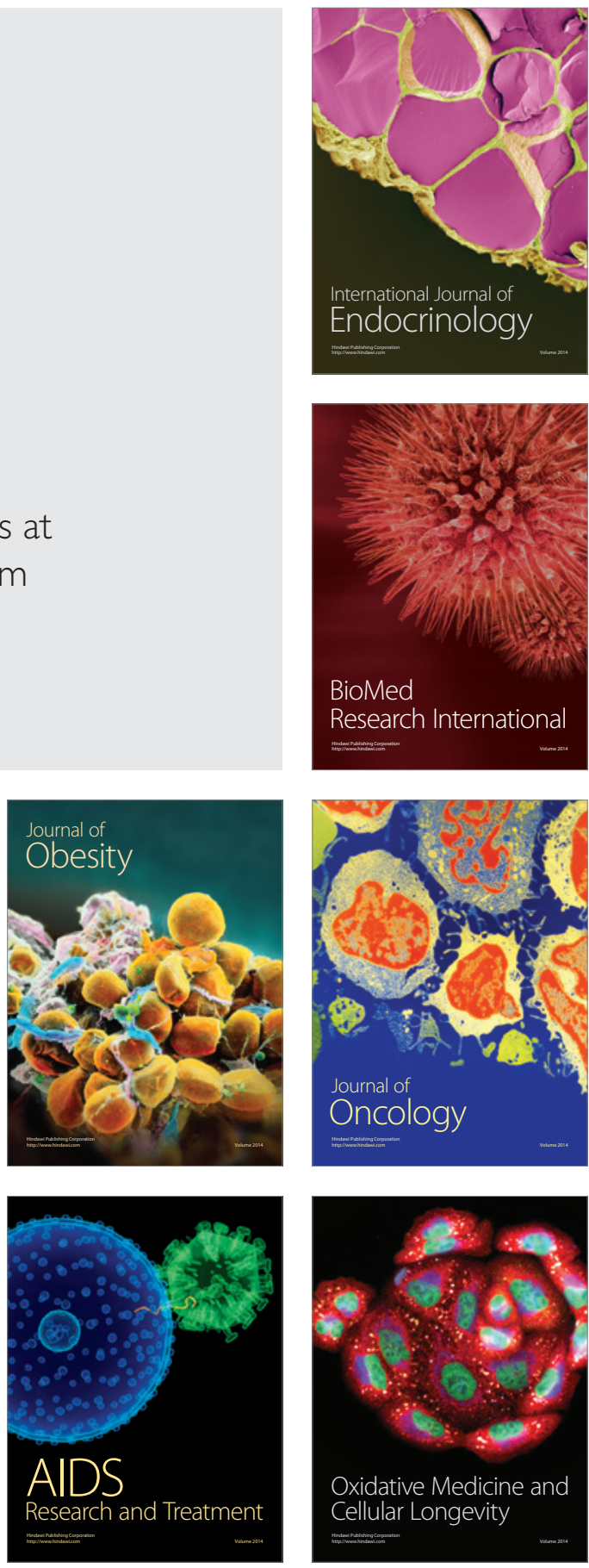